\title{
Avaliação ambiental do parque urbano Chico Mendes, Porto Alegre - RS, Brasil
}

\author{
Environmental assessment of the urban Park Chico Mendes, Porto Alegre - RS, Brazil
}

\author{
Vladimir Stolzenberg Torres $^{1 *}$, Felipe Todeschini ${ }^{2}$ e Mariana Freitas Farias ${ }^{3}$
}

\author{
${ }^{1}$ Secretaria Municipal de Meio Ambiente de Porto Alegre. Doutor \\ ${ }^{2}$ Pontifícia Universidade Católica do Rio Grande do Sul. Acadêmico de Ciências Biológicas e Estagiário da \\ Secretaria Municipal do Meio Ambiente de Porto Alegre \\ ${ }^{3}$ Universidade do Vale do Rio dos Sinos. Acadêmica de Ciências Biológicas e Estagiária da Secretaria Municipal \\ do Meio Ambiente de Porto Alegre
}

\begin{abstract}
Resumo
Os impactos ambientais nesse século XXI aumentaram perigosamente e com isto foi reforçado o paradigma da manutenção do ambiente. No âmbito de uma floresta urbana, passou-se a preconizar o aumento de áreas verdes e a conectividade das mesmas, além do incentivo ao maior uso de essências nativas, bem como, uma ampliação na diversidade de espécies. O objetivo do presente estudo foi diagnosticar as condições ambientais do Parque Municipal Chico Mendes. A análise qualitativa da avifauna e da flora baseou-se na presença e na ausência comparativamente a outros estudos. Foi estimado o Índice de Sørensen para flora e para avifauna, e foi realizada uma avaliação das condições fitossanitárias dos espécimes arbóreos. Assim, no presente estudo, foram catalogadas 51 espécies de aves no Parque Chico Mendes, sendo 23 consideradas como migratórias e 28 como residentes, duas espécies de anfíbios, 10 de répteis, nove de mamíferos e 55 de plantas. O indice de Sørensen para avifauna expressou um valor de similaridade de 0,65420 quando comparado a outros estudos; enquanto que o mesmo índice, para flora arbórea ficou na ordem de 0,37500. É necessário o desenvolvimento de ferramentas de sensibilização e transformação que promovam mudanças de conduta na comunidade do entorno.
\end{abstract}

Palavras-chave: Impactos ambientais. Análise qualitativa. Sensibilização ambiental

\begin{abstract}
The environmental impacts in this XXI century increased dangerously and, thus, was reinforced the paradigm of maintaining the environment. In the context of an urban forest, was recommended the increase of green areas and the connectivity between them, as well as encourage greater use of native essences, even as an expansion in the diversity of species. The aim of this study was to diagnose the environmental conditions of Chico Mendes Municipal Park. The qualitative analysis of avifauna and plant was based on the presence and absence compared to other studies. The Sørensen index was estimated for flora and avifauna, and a review of the phytosanitary conditions of tree specimens. Thus, in the present study were cataloged 51 species of birds in the Chico Mendes Park, being 23 considered migratory and 28 as residents, 2 species of amphibians, reptiles 10, 9 mammals and 55 plants. The Sørensen index for avifauna expressed a similarity value of 0.65420 when compared to other studies; while the same index for tree flora was in order of 0.37500. It is necessary the development of awareness and transformation tools that promote behavioral change in the surrounding community.
\end{abstract}

Keywords: Environmental impacts. Qualitative analysis. Environmental awareness 


\section{Introdução}

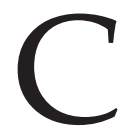

onforme Lombardo (1990), o ambiente urbano é a resultante das interações dos fatores ambientais, biológicos e socioeconômicos, em que o meio edificado pelo homem predomina sobre o meio físico, causando profundas alterações sobre este e na qualidade de vida dos seres.

Os impactos ambientais associados ao processo de urbanização foram ampliados perigosamente neste último século. A expansão periférica das cidades brasileiras e a forma precária como foram implantados os novos assentamentos criaram um quadro de grave degradação. No atual estágio da situação econômica e do planejamento urbano, evitar a expansão física das cidades está fora do alcance das políticas urbanas, mas cabe perfeitamente buscar formas de prevenir novos impactos sócio-ambientais.

Desta forma, a manutenção, o aumento de áreas e a interligação das áreas verdes urbanas e também na vizinhança imediata de uma cidade são fundamentais, usando-se para isso, de preferência, espécies nativas e um maior número de espécies, visando diversificar também as fontes de alimentos.

A importância de áreas verdes urbanas vem sendo ressaltada em vários trabalhos. Os parques urbanos são áreas verdes que apresentam um grande potencial para a conservação da biodiversidade nas cidades, pois oferecem áreas de abrigo e de forrageamento para muitas espécies animais, influenciam no microclima local, além da função social, pois elas proporcionam o primeiro contato com o ambiente natural de muitas pessoas, atuando como forma de resgate da memória dos biomas nativos ao local agora urbanizado (ISERNHAGEN et al., 2009). Além disto, conforme Macedo e Sakata (2003), os parques urbanos podem formar mosaicos ao longo do território, nos chamados Corredores Ecológicos, trazendo benefícios não somente para a biodiversidade, mas também para a conservação do solo e dos recursos hídricos, além dos benefícios intangíveis como aqueles associados à contemplação da paisagem.

De outra perspectiva, conforme Japyassú e Brescovit (2006), o adensamento urbano leva à formação de inúmeros microecossistemas, os quais refletem diretamente na fauna urbana, tornando-a uma resultante de fatores tanto ecológicos como históricos e culturais; com isto, sendo não originada exclusivamente pela degradação da composição faunística original através do processo de urbanização, mas também pela constante introdução de espécies consideradas alóctones àquela região considerada.

Conforme Guzzo (2006), as áreas verdes contribuem para a qualidade de vida através da valoração ecológica e humanística evidenciando-se, por conseguinte, que a criação e manutenção de parques urbanos vêm ao encontro dos problemas levantados, assim norteando o objetivo precípuo do presente estudo, qual seja, diag- nosticar as condições ambientais do Parque Municipal Chico Mendes.

\section{Materiais e métodos}

O Parque Municipal Chico Mendes localiza-se no município de Porto Alegre-RS, sendo delimitado pelas ruas José Pereira de Borba, Sargento Silvio Delmar Hollenbach, Martin Felix Berta, Alceri Garcia Flores, Millo Raffin e Irmão Ildefonso Luiz no contexto do bairro Mário Quintana. Possui uma área total de 25,3ha, caracterizada pela presença de campos, áreas de mata nativa e áreas de eucaliptais entremeadas com mata secundária. Sua localização, acrescida da presença de uma ocupação residencial irregular em seu interior, determina fortes interferências antrópicas na paisagem do mesmo.

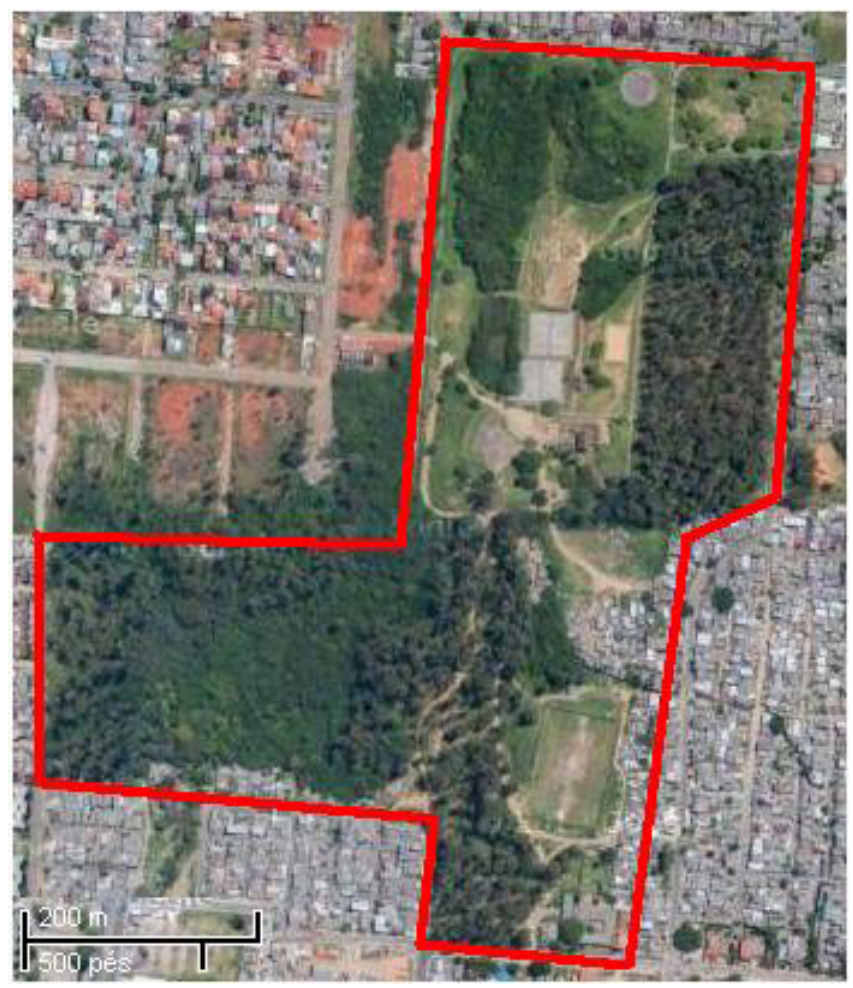

Figura 1. Delimitação do Parque Municipal Chico Mendes, Porto Alegre, RS (Fonte: Imagens (02014 CNES / Astrium, DigitalGlobe).

O inventário florístico foi realizado ao longo dos anos de 2011 a 2014, através de avaliação in loco, com a identificação taxonômica sendo realizada com base em Longhi (1995), Lorenzi e Souza (1995), Marchiori (1996), Backes e Irgang (2002) Lorenzi (2002), Lorenzi et al. (2003), Backes e Irgang (2004), Sobral et al. (2006), Pacheco e Marques (2006), e Gomes (2011); e posteriormente com a revisão processada através do Missouri Botanical Garden <http://www.tropicos.org/>. O levantamento faunístico, por sua vez, foi realizado a partir de 2013, com a identificação taxonômica sendo realizada com 
base em Silva (1994) Efe e Mohr (1999), Efe et al. (2001), Bujes (2010), Penter et al. (2008), Endrigo (2010), Witt (2013); e posteriormente com a revisão nomenclatural da avifauna processada através do Avibase - the world bird database <http://avibase.bsc-eoc.org/avibase.jsp $>$ e a dos demais grupos através do Integrated Taxonomic Information System <http://www.itis.gov/>.

Para a análise qualitativa da avifauna e da flora arbórea/arbustiva, baseada em presença e ausência, foram geradas listas de espécies observadas; sendo a lista de avifauna comparada com os dados de Efe et al. (2001) e lista de flora comparada com os dados de Torres et al. (comunicação pessoal) quanto à similaridade pelo Índice de Sørensen (KENT; COKER, 1992), que dá peso dois para as espécies comuns que ocorrem nas amostras analisadas, calculado pela fórmula:

$$
S=2 a /(2 a+b+c)
$$

Onde a é o número de espécies comuns em dois períodos tomados para a comparação, b é o número de espécies exclusivas registrados no período 1 e c é o número de espécies exclusivas registrados no período 2.

Para determinar o grau de hemiparasitismo (organismos que, ao contrário dos holoparasitas, não dependem inteiramente do seu hospedeiro para sobreviver), foi empregado o sistema de Oliveira e Kappel (1994) assim descrito: baixo, quando a incidência de hemiparasitas era inferior a $25 \%$ da copa do hospedeiro; média, quando a partir de $25 \%$ e inferior a $50 \%$; e alta, quando igual a $50 \%$ ou mais.

\section{Resultados e discussão}

No âmbito de inventariamento, os resultados encontram-se expressos através da Tabelas 1-3, com sua análise sendo realizada seqüencialmente às mesmas.

Tabela 1 - Avifauna avistada no âmbito do Parque Municipal Chico Mendes

\begin{tabular}{|c|c|c|c|}
\hline Nome popular & Nome científico & Residente & Migratório \\
\hline Alma-de-gato & Piaya cayana Linnaeus, 1766 & & $\mathrm{X}$ \\
\hline Andorinha-do-campo & Progne tapera Linnaeus, 1766 & & $X$ \\
\hline Andorinha-do-temporal & Chaetura andrei Berlepsch \& Hartert, 1902 & & $X$ \\
\hline Andorinha-pequena-de-casa & Pygochelidon cyanoleuca Vieillot, 1817 & & $X$ \\
\hline Anu-branco & Guira guira (Gmelin, 1788) & $X$ & \\
\hline Anú-preto & Crotophaga ani Linnaeus, 1758 & & $X$ \\
\hline Arredio-oliváceo & Cranioleuca obsoleta (Reichenbach, 1853) & & $X$ \\
\hline Asa-de-telha & Agelaioides badius (Vieillot, 1819) & & $X$ \\
\hline Bacurau & Hydropsalis torquata (Gmelin, 1789) & $X$ & \\
\hline Beija-flor & Heliomaster furcifer (Shaw, 1812) & $X$ & \\
\hline Bem-te-vi & Pitangus sulphuratus (Linnaeus, 1766) & $X$ & \\
\hline Bico-de-lacre & Estrilda astrild (Linnaeus, 1758) & $X$ & \\
\hline Cambacica & Coereba flaveola (Linnaeus, 1758) & $X$ & \\
\hline Canário-da-terra & Sicalis flaveola (Linnaeus, 1766) & $X$ & \\
\hline Cardeal & Paroaria coronata (Miller, 1776) & $\mathrm{X}^{1}$ & \\
\hline Caturrita & Myiopsitta monachus (Boddaert, 1783) & $X$ & \\
\hline Chimango & Milvago chimango (Vieillot, 1816) & & $X$ \\
\hline Corruíra & Troglodytes musculus Naumann, 1823 & $X$ & \\
\hline Galinhola & Gallinula chloropus (Linnaeus, 1758) & & $X$ \\
\hline Garça-branca-pequena & Egretta thula (Molina, 1782) & & $X$ \\
\hline Garibaldi & Chrysomus ruficapillus (Vieillot, 1819) & & $X$ \\
\hline Gavião-carcará & Polyborus plancus (Miller, 1777) & & $X$ \\
\hline Gavião-carijó & Buteo magnirostris (Gmelin, 1788) & & $X$ \\
\hline Jaçanã & Jacana jaçanã (Linnaeus, 1766) & & $X$ \\
\hline João-de-barro & Furnarius rufus (Gmelin, 1788) & $x$ & \\
\hline
\end{tabular}


Tabela 1 - Avifauna avistada no âmbito do Parque Municipal Chico Mendes, Continuação ...

\begin{tabular}{l|l|c|c}
\hline \multicolumn{1}{c|}{ Nome popular } & \multicolumn{1}{|c|}{ Nome científico } & Residente & Migratório \\
\hline Juriti & Leptotila verreauxi Bonaparte, 1855 & $\mathrm{X}$ & \\
\hline Maçarico-de-cara-pelada & Phimosus infuscatus (Lichtenstein, 1823) & $\mathrm{X}$ & \\
\hline Mariquita & Setophaga pitiayumi (Vieillot, 1817) & $\mathrm{X}$ & \\
\hline Pardal & Passer domesticus (Linnaeus, 1758) & $\mathrm{X}$ & \\
\hline Pia-cobra & Geothlypis aequinoctialis (Gmelin, 1789) & & $\mathrm{X}$ \\
\hline Pica-pau-do-topete-vermelho & Campephilus melanoleucos (Gmelin, 1788) & $\mathrm{X}$ & \\
\hline Pica-pau-verde-barrado & Colaptes melanochloros (Gmelin, 1788) & $\mathrm{X}$ & \\
\hline Pintassilgo-de-cabeça-preta & Sporagra magellanica (Vieillot, 1805) & $\mathrm{X}$ & \\
\hline Pombo-doméstico & Columba lívia Gmelin, JF, 1789 & $\mathrm{X}$ & \\
\hline Quero- quero & Vanellus chilensis (Molina, 1782) & $\mathrm{X}$ & \\
\hline Quete & Poospiza lateralis (Nordmann, 1835) & & $\mathrm{X}$ \\
\hline Quiriquiri & Falco sparverius Linnaeus, 1758 & & $\mathrm{X}$ \\
\hline Rolinha-picuí & Columbina picui (Temminck, 1813) & $\mathrm{X}$ & \\
\hline Rolinha-roxa & Columbina talpacotii (Temminck, 1810) & $\mathrm{X}$ & \\
\hline Sabiá-do-campo & Mimus saturninus (Lichtenstein, 1823) & & $\mathrm{X}$ \\
\hline Sabiá-laranjeira & Turdus rufiventris Vieillot, 1818 & $\mathrm{X}$ & \\
\hline Sabiá-poca & Turdus amaurochalinus Cabanis, 1850 & $\mathrm{X}$ & \\
\hline Sanhaçu & Thraupis sayaca (Linnaeus, 1766) & & $\mathrm{X}$ \\
\hline Sanhaçu-papa-laranja & Thraupis bonariensis (Gmelin, 1789) & & $\mathrm{X}$ \\
\hline Saracura-do-banhado & Pardirallus sanguinolentus (Swainson, 1838) & $\mathrm{X}$ & \\
\hline Savacu & Nycticorax nycticorax (Linnaeus, 1758) & & $\mathrm{X}$ \\
\hline Suiriri & Tyrannus melancholicus Vieillot, 1819 & $\mathrm{X}$ & \\
\hline Tesourinha & Tyrannus savana Vieillot, 1808 & & $\mathrm{X}$ \\
\hline Tico-tico & Zonotrichia capensis (Statius-Müller, 1776) & $\mathrm{X}$ & \\
\hline Urubu-da-cabeça-preta & Coragyps atratus (Bechstein, 1793) & & $\mathrm{X}$ \\
\hline Vira-bosta & Molothrus bonariensis (Gmelin, 1789) & & $\mathrm{X}$ \\
\hline
\end{tabular}

${ }^{1}$ Exemplares observados no âmbito do Parque, que não podem ser considerados como residentes e não possuem hábitos migratórios, sendo considerada sua presença em face de soltura ou fuga de cativeiro

Segundo Bortoleto (2004), a presença de avifauna e a formação de ninhos por pássaros em árvores urbanas representa um dos principais indicadores de qualidade de vida e ambiental nas cidades. Assim, no presente estudo, foram catalogadas 51 espécies de aves no Parque Chico Mendes, sendo 23 consideradas como migratórias e 28 como residentes da região, diferindo, portanto, do estudo de Efe e Mohr (1999), os quais apontaram a presença de 72 espécies de aves. Porém, um segundo estudo realizado por Efe et al. (2001) reduziu esta quantidade para 56 espécies, valor este que mais se aproxima dos resultados ora obtidos no presente estudo.

É interessante considerar que, em um espaço temporal de apenas dois anos entre os dois estudos levados a cabo por Efe (EFE; MOHR, 1999; e EFE et al., 2001), houve um declínio na ordem de 16 espécies. De outra feita, o inventário de Efe et al. (2001) possui 21 espécies que não foram observadas no presente estudo, assim como foram encontradas 16 espécies no âmbito do parque que não são conhecidas pelo estudo daqueles autores. Fica evidente um universo de 37 espécies que representam uma divergência de observações entre este último estudo e o presente.

Porém, a aplicação do Índice de Sørensen (Diversidade $\beta$ ) para comparar os registros obtidos neste estudo com os de Efe et al. (2001), expressou um valor de similaridade na ordem de 0,65420 que pode ser considerado como mediano. Esse índice estuda as causas e efeitos da co-habitação de espécies num determinado ambiente, assim como dos processos correlacionados em sua continuidade ou em mudança ao longo do tempo. Sua desvantagem, entretanto, está em não considerar a abundância das espécies detectadas, o que muito provavelmente ocasionaria a sua redução.

Segundo Filho e Medeiros (2006), a vegetação nas áreas urbanas desempenha importante função ecológica, 
uma vez que estes locais se convertem em importantes ambientes de abrigo, descanso, nidificação e fonte de alimentação para as aves. Pode-se verificar neste estudo que a maioria das espécies identificadas apresenta hábitos alimentares generalistas, sendo estas extremamente beneficiadas no ambiente urbano, uma vez que elas podem encontrar alimento ao longo de todo o ano, independentemente das espécies vegetais em frutificação. Além disso, conforme Silva et al. (2011), é muito provável que algumas das espécies que freqüentam o parque fazem do local um refúgio, já que, com a destruição dos seus habitat's naturais, podem ser forçadas a se realocarem em outras regiões, como por exemplo, em parques urbanos.

Tabela 2 - Fauna (exceto aves) observada no âmbito do Parque Municipal Chico Mendes

\begin{tabular}{|c|c|c|c|c|}
\hline Grupo & Nome popular & Nome científico & Autóctone & Introduzido \\
\hline \multirow{2}{*}{ Amphibia } & Perereca-do-banhado & $\begin{array}{l}\text { Hypsiboas pulchellus } \\
\text { (Duméril \& Bibron, 1841) }\end{array}$ & $X$ & \\
\hline & Sapinho-de-jardim & $\begin{array}{l}\text { Rhinella fernandezae } \\
\text { (Gallardo,1957) }\end{array}$ & $X$ & \\
\hline \multirow{10}{*}{ Reptilia } & Cipó-listrada & $\begin{array}{l}\text { Philodryas olfersii } \\
\text { (Lichtenstein,1823) }\end{array}$ & $X$ & \\
\hline & Cobra-cega & $\begin{array}{l}\text { Amphisbaena trachura } \\
\text { Cope, } 1885\end{array}$ & $X$ & \\
\hline & Cobra-cega & $\begin{array}{l}\text { Amphisbaena prunicolor } \\
\text { (Cope, 1885) }\end{array}$ & $X$ & \\
\hline & Cobra-cega & $\begin{array}{l}\text { Amphisbaena darwinii } \\
\text { Burmeister, } 1861\end{array}$ & $x$ & \\
\hline & Falsa-coral & $\begin{array}{l}\text { Oxyrhopus rhombifer Duméril, } \\
\text { (Bibron \& Duméril, 1854) }\end{array}$ & $X$ & \\
\hline & Lagartinho-verde & $\begin{array}{l}\text { Teius oculatus } \\
\text { (D'Orbigny \& Bibron,1837) }\end{array}$ & $X$ & \\
\hline & Lagartixa & $\begin{array}{l}\text { Hemidactylus mabouia } \\
\text { Moreau de Jonnès, } 1818\end{array}$ & & $X$ \\
\hline & Cágado-preto & $\begin{array}{l}\text { Acanthochelys spixii } \\
\text { (Duméril \& Bibron, 1835) }\end{array}$ & & $X$ \\
\hline & Tartaruga-tigre-da-água & $\begin{array}{l}\text { Trachemys dorbigni } \\
\text { (Duméril \& Bibron, 1835) }\end{array}$ & $X$ & \\
\hline & $\begin{array}{l}\text { Tarturuga-de-orelha- } \\
\text { vermelha }\end{array}$ & $\begin{array}{l}\text { Trachemys scripta elegans } \\
\text { (Wied, 1839) }\end{array}$ & & $X$ \\
\hline \multirow{9}{*}{ Mammalia } & Cachorro & $\begin{array}{l}\text { Canis familiaris } \\
\text { Linnaeus, } 1758\end{array}$ & & $X$ \\
\hline & Camundongo & $\begin{array}{l}\text { Mus musculus } \\
\text { Linnaeus, } 1758\end{array}$ & & $X$ \\
\hline & Gambá-de-orelha-branca & $\begin{array}{l}\text { Didelphis albiventris } \\
\text { Lund } 1840\end{array}$ & $X$ & \\
\hline & Gato-doméstico & $\begin{array}{l}\text { Felis catus } \\
\text { Linnaeus, } 1758\end{array}$ & & $\mathrm{X}^{1}$ \\
\hline & Morcego-das-frutas & $\begin{array}{l}\text { Artibeus lituratus } \\
\text { (Olfers, 1818) }\end{array}$ & $X$ & \\
\hline & Morcego-das-casas & $\begin{array}{l}\text { Tadarida brasiliensis } \\
\text { (L. Geoffroy, 1824) }\end{array}$ & $X$ & \\
\hline & Preá & $\begin{array}{l}\text { Cavia aperea } \\
\text { Exerleben, } 1777\end{array}$ & $X$ & \\
\hline & Ratazana & $\begin{array}{l}\text { Rattus norvegicus } \\
\text { (Berkenhout, 1769) }\end{array}$ & & $X$ \\
\hline & Rato-comum-das-casas & $\begin{array}{l}\text { Rattus rattus } \\
\text { (Linnaeus, 1758) }\end{array}$ & & $X$ \\
\hline
\end{tabular}


Apesar das alterações e impactos ambientais caracterizando-se em uma área com forte interferência antrópica, localizado em meio da malha urbana da cidade, com o inventário de mamíferos na sua maioria composta por espécies generalistas, o Parque Chico Mendes ainda apresenta uma riqueza de espécies que não pode deixar de ser considerada, especialmente quando se considera esta área uma significativa representação da floresta urbana neste setor da cidade.

Segundo Young et al. (2003), um levantamento rápido, por ser de curta duração, produz uma lista incompleta de espécies, pois qualquer lista de fauna requer anos de amostragem e uma grande variedade de técnicas. Com isso, apesar de terem sido utilizadas variadas técnicas de observação neste estudo, existe a possibilidade de que a lista de espécies registradas ainda esteja subestimada. Nesse sentido, visualizações e vestígios são registros eventuais, extremamente dependentes de fatores espaciais e temporais e, portanto, nem sempre retratam o número real de espécies ocorrentes.

O baixo número de anfíbios registrado pode estar associado ao inadequado esforço de captura, determinando, com isto, a não efetiva observação de outras espécies que possam ocorrer na área. Semelhantemente, encontrase a situação de Reptilia, muito embora para o gênero Amphisbaena Linnaeus, 1758 os registros obtidos sejam consoantes com os de Torres (2003), suportados ainda pela revisão taxonômica realizada por Maciel (2011). No que tange à Chelonia, os resultados denotam afinidade com os dados obtidos por Fabres et al. (s/d) para o Parque Moinho de Vento, considerando-se que o volume de freqüentadores daquele parque é significativamente maior do que o Chico Mendes e, consequentemente, há uma intervenção antrópica muito maior, uma vez que nenhuma das espécies de tartarugas e cágados (mesmo as nativas) possuem ocorrência natural no local, tendo sido indevidamente introduzidas em algum momento ao longo da história do mesmo. Da mesma forma, "solturas" indevidas, com maior probabilidade, no âmbito do Parque Chico Mendes devem ocorrer nos dois arroios que o cortam, por conseguinte não permanecendo na área.

As interações ecológicas que se estabelecem entre as espécies de mamíferos são muito importantes para a conservação de várias outras espécies de animais e plantas, principalmente em ambientes fragmentados. As espécies frugívoras e herbívoras, como os gambás, morcegos e roedores registrados neste estudo, desempenham papel muito importante na manutenção da diversidade de árvores da floresta através da dispersão e predação de sementes e de plântulas (WRIGHT et al. 2000; PARDINI et al. 2003). Neste sentido, a captura de um exemplar de Artibeus lituratus (OLFERS, 1818) confere a esta espécie uma importância provável na dispersão de várias espécies vegetais no Parque Chico Mendes, conforme Menegat et al. (1998).

Apesar da identificação de apenas duas espécies de morcegos (sendo uma frugívora e outra insetívora), é fundamental considerar que, conforme Borges-Filho et al. (2011), os parques urbanos são importantes para a ecologia destes animais, uma vez que, a manutenção de parte da vegetação nativa original da região proporciona um aumento potencial na disponibilidade de recursos para os mesmos. Associe-se a este aspecto certo grau de impacto antrópico que pode se revelar igualmente favorável em face de intervenções que se tornem geradoras de maiores oportunidades alimentares, e obtêm-se então contribuições para o estabelecimento de populações na região.

A presença de cães e gatos domésticos no Parque Chico Mendes é um fato previsível devido à proximidade com zonas urbanizadas. Estudos sobre o impacto de gatos domésticos sobre a fauna silvestre são muito freqüentes, já o mesmo não é verificado para os cães. Woods et al. (2003) chamam atenção ao fato de que parte da população de gatos é alimentada por pessoas e, portanto, não é regulada pela disponibilidade de presas silvestres. Para Baker et al. (2003), o decréscimo das populações animais silvestres está relacionado à predação por gatos domésticos, tanto quanto à redução e fragmentação de hábitat. Por outro lado, Lepczyk et al. (2003) citam que os gatos domésticos ocupam o papel dos predadores naturais, gerando competição por recursos. De qualquer maneira, é provável que exista uma forte relação entre a extinção de espécies nativas pela introdução de espécies exóticas (BURBRIDGE; MANLY, 2002).

As fortes alterações que se verificam em meio urbano são motivadas pela atividade humana, e com isto, diferentes associações de espécies florestais determinam diferentes condições ambientais para cada local. Caso o ambiente sofra modificações, as associações vegetais também se modificam e adaptam-se às novas condições. De acordo com Schneider (1993), estas alterações normalmente resultam em um empobrecimento da qualidade da floresta, o que não se confirma no contexto da floresta urbana em face ao seu elevado equilíbrio dinâmico, conforme Puente et al. (2005).

O Parque Chico Mendes apresenta uma flora bastante heterogênea, com 55 espécies identificadas neste primeiro estudo. Porém, é fundamental estabelecer que nos que se antecedem a sua implantação em 1991, tratava-se de área com extrativismo vegetal para comercialização de lenha. Assim, boa parte da vegetação que ora se observa no âmbito do Parque, constitui-se em mata secundária ainda em recuperação, porém ainda assim expressando interferências antrópicas, seja pelo plantio, seja pela remoção clandestina de espécimes.

É salutar o registro de alguns raros exemplares de Dicksonia sellowiana Hook., bem como de Erythrina crista-galli L. No que tange à primeira espécie, contava-se exclusivamente com um único registro para Porto Alegre, realizado por Senna e Kazmirczak (1997). É considerada espécie em perigo pela Portaria № 37-N/1992, do IBAMA. 
Tabela 3. Lista dos representantes da flora amostradas no parque Chico Mendes.

\begin{tabular}{|c|c|c|c|c|}
\hline Nome popular & Espécie & Hábito $^{1}$ & Autóctone & Alóctone \\
\hline Abacate & Persea americana Mill. & Arbóreo & & $X$ \\
\hline Açoita-cavalo & Luehea divaricata Mart. & Arbóreo & $x$ & \\
\hline Amoreira & Morus nigra L. & Arbóreo & & $x$ \\
\hline Angico-vermelho & Parapiptadenia rigida (Benth.) Brenan & Arbóreo & $x$ & \\
\hline Araçazeiro & Psidium cattleianum Sabine & Arbóreo & $x$ & \\
\hline Araucária & $\begin{array}{l}\text { Araucaria angustifolia } \\
\text { (Bert.) O. Kuntze }\end{array}$ & Arbóreo & $X$ & \\
\hline Aroeira-periquita & Schinus molle L. & Arbóreo & $x$ & \\
\hline Aroeira-vermelha & Schinus terebinthifolia Raddi & Arbóreo & $x$ & \\
\hline Astrapeia & $\begin{array}{l}\text { Dombeya wallichii } \\
\text { (Lindl.) Benth. ex Baill. }\end{array}$ & Arbustivo & & $X$ \\
\hline Bananeira & Musa x paradisíaca L. & Cespitoso & & $X$ \\
\hline Butiazeiro & $\begin{array}{l}\text { Butia aff. eriospatha } \\
\text { (Mart. ex Drude) Becc. }\end{array}$ & Arbóreo & $X$ & \\
\hline Caliandra-rosa & Calliandra brevipes Benth. & Arbustivo & $x$ & \\
\hline Caliandra-vermelha & Calliandra tweedii Benth. & Arbustivo & $X$ & \\
\hline Canafístula & Peltophorum dobium (Spreng.) Taub. & Arbóreo & $X$ & \\
\hline Canela-amarela & Nectandra lanceolata Nees & Arbóreo & $x$ & \\
\hline Canela-branca & Ocotea spixiana (Ness) Mez & Arbustivo & & \\
\hline Capororoca & Myrsine ferruginea (Sw.) R.Br. & Arbustivo & $X$ & \\
\hline Cedro & Cedrela fissilis Vell. & Arbóreo & $x$ & \\
\hline Cerejeira & Eugenia involucrata DC. & Arbóreo & $x$ & \\
\hline Chá-de-bugre & Casearia punctata Spreng. & Arbóreo & $x$ & \\
\hline Chal-chal & $\begin{array}{l}\text { Allophylus edulis } \\
\text { (A. St.-Hill. Cambess. \& A. Juss.) } \\
\text { Radlk }\end{array}$ & Arbóreo & $X$ & \\
\hline Cinamomo & Melia azedarach L. & Arbóreo & & $X$ \\
\hline Cocão & $\begin{array}{l}\text { Erythroxylum argentinum } \\
\text { O.E. Schulz }\end{array}$ & Arbóreo & $X$ & \\
\hline Corticeira-do-banhado & Erythrina crista-galli L. & Arbóreo & $X$ & \\
\hline Eucalipto & Eucalyptus grandis W. Hill ex Maid. & Arbóreo & & $X$ \\
\hline
\end{tabular}


Tabela 3. Lista dos representantes da flora amostradas no parque Chico Mendes. Continuação...

\begin{tabular}{|c|c|c|c|c|}
\hline Nome popular & Espécie & Hábito $^{1}$ & Autóctone & Alóctone \\
\hline Eucalipto & saligna Sm. & Arbóreo & & $x$ \\
\hline Eucalipto cascudo & Eucalyptus robusta Sm. & Arbóreo & & $x$ \\
\hline Figueira-da-folha-miúda & Ficus cestrifolia Schott ex Spreng. & Arbóreo & $x$ & \\
\hline Figueira-de-vaso & Ficus benjamina L. & Arbóreo & & $x$ \\
\hline Grandiúva & Celtis canescens Kunth & Arbóreo & $x$ & \\
\hline Guapuruvu & $\begin{array}{l}\text { Schizolobium parahyba (Vell.) S. F. } \\
\text { Blake }\end{array}$ & Arbóreo & $x$ & \\
\hline Hibisco & Hibiscus rosa-sinensis $\mathrm{L}$. & Arbustivo & & $X$ \\
\hline Ingá-feijão & Inga marginata Wild. & Arbóreo & $x$ & \\
\hline Ipê-amarelo & $\begin{array}{l}\text { Handroanthus chrysotrichus (Mart. } \\
\text { ex A. DC.) Mattos }\end{array}$ & Arbóreo & $x$ & \\
\hline Ipê-roxo & $\begin{array}{l}\text { Handroanthus avellanedae (Lorentz } \\
\text { ex Griseb.) Mattos }\end{array}$ & Arbóreo & $x$ & \\
\hline Jacarandá & Jacaranda mimosifolia D. Don. & Arbóreo & & $x$ \\
\hline Jambolão & Syzygium cumini (L.) Skeels & Arbóreo & & $X$ \\
\hline Jasmim-amarelo & Jasminum mesnyi Hance & Arbustivo & & $x$ \\
\hline Jerivá & $\begin{array}{ll}\text { Syagrus romanzoffiana } & \text { (Cham.) } \\
\text { Glassman } & \\
\end{array}$ & Arbóreo & & $x$ \\
\hline Leiteiro & Sapium glandulatum (Vell.) Pax & Arbóreo & $x$ & \\
\hline Limão-bergamota & Citrus x limon Burm. f. & Arbóreo & & $x$ \\
\hline Malvavisco & Malvaviscus arboreus Cav. & Arbustivo & & $x$ \\
\hline Mamica-de-cadela & Zanthoxylum rhoifolium Lam. & Arbóreo & $x$ & \\
\hline Maricá & Mimosa bimucronata (DC.) Kuntze & Arbóreo & $x$ & \\
\hline Paineira & Ceiba speciosa A. St.-Hill. & Arbóreo & $x$ & \\
\hline Pata-de-vaca & Bauhnia forficata Link & Arbóreo & $x$ & \\
\hline Pitangueira & Eugenia uniflora L. & Arbustivo & $x$ & \\
\hline Pseudo bombax & $\begin{array}{l}\text { Pseudobombax grandiflorum (Cav.) } \\
\text { A. Robyns }\end{array}$ & Arbóreo & & $x$ \\
\hline Salso-chorão & Salix babylonica $\mathrm{L}$. & Arbóreo & $x$ & \\
\hline Taquareira-listrada & $\begin{array}{l}\text { Bambusa vulgaris Schrad. ex J. C. } \\
\text { Wendl. }\end{array}$ & Cespitoso & & $x$ \\
\hline
\end{tabular}


Tabela 3. Lista dos representantes da flora amostradas no parque Chico Mendes. Continuação...

\begin{tabular}{|c|c|c|c|c|}
\hline Nome popular & Espécie & Hábito $^{1}$ & Autóctone & Alóctone \\
\hline Taquareira-nativa & Merostachys speciosa Spreng. & Cespitoso & $x$ & \\
\hline Timbaúva & $\begin{array}{l}\text { Enterolobium contortisiliquum (Vell.) } \\
\text { Morong }\end{array}$ & Arbóreo & $x$ & \\
\hline Tipuana & Tipuana tipu (Benth.) Kuntze & Arbóreo & & $X$ \\
\hline Vassoura-vermelha & Dodonaea viscosa Jacq. & Arbustivo & $x$ & \\
\hline Xaxim & Dicksonia sellowiana Hook. & Arborescente & $X$ & \\
\hline
\end{tabular}

A segunda, semelhantemente, é considerada planta imune ao corte no Rio Grande do Sul pela Lei Estadual 9.519/92 (Art. 33).

Torres et al. (comunicação pessoal) encontraram, no conjunto de dois fragmentos de Porto Alegre, 41 espécies arbóreas, resultado este que difere daquele encontrado no presente estudo, sugerindo que o Parque possa ter sofrido maior interferência antrópica no que se refere à inclusão de novas espécies do que aqueles fragmentos, ou, alternativamente, tenha conservado maior nível de diversidade de espécies, do conjunto vegetal original que o constituía. Assim, aplicando-se o Índice de Sørensen (Diversidade $\beta$ ) para comparar os registros obtidos neste estudo com os dos fragmentos unificados de Torres et al. (comunicação pessoal), obtém-se um valor de similaridade na ordem de 0,37500 que pode ser considerado baixo. De forma geral, dentre as espécies consideradas comuns a ambos os ambientes, prevalecem aquelas que seriam consideradas como alóctones de forma geral, embora se percebam, evidentemente, algumas exceções.

Do ponto de vista fitossanitário, no âmbito do parque, se sobressaem os problemas de ervas-de-passarinho que acometem, particularmente, as espécies de eucaliptos. Como regra geral, são consideradas como importantes pragas, porque afetam a qualidade da arborização urbana. Apesar disto, apresentam elevada importância nos ecossistemas naturais (ou artificiais, considerando-se o urbano) como fonte de alimentos para as aves, conforme Cazetta e Galetti (2003), o que fica plenamente demonstrado pela alta dispersão. Ainda segundo Cazetta e Galetti (op. cit.), árvores com alto grau de infestação por erva-de-passarinho são mais predispostas ao ataque de insetos e mais susceptíveis a estresses ambientais do que indivíduos saudáveis da mesma espécie, o que, no contexto do parque, parece ser respaldado pela evidência de uma maior incidência de cupins em espécimes com elevada infestação.

Para Eucalyptus spp, a incidência foi considerada de média a alta, registrando-se a presença de exemplares mortos por conta da elevada infestação. Foram observadas infestações em grau considerado baixo em Salix babylonica L. e Syzygium cumini (L.) Skeels. Tais resultados demonstram claramente que existem alguns aspectos característicos na relação entre parasita/hospedeiro. Certas espécies arbóreas, por exemplo, podem ter atributos que as tornem mais susceptíveis do que outras, apresentando, consequentemente, um alto índice de infestação. Leal et al. (2006) citam que a infestação está diretamente relacionada à espécie do hospedeiro, já que algumas se apresentam bastante sensíveis, enquanto outras podem ser tolerantes ou até mesmo resistentes.

Outros problemas, entretanto, acometem a sanidade de alguns espécimes arbóreos localizados em áreas do parque sob influência do regime hídrico do arroio que corta a área interna pela parte oeste. A exemplo do arroio situado mais a leste, este serve de local para despejo de efluentes "in natura" provenientes desta região da cidade, além da deposição irregular de lixo em seu interior. Essa condição, além de ser desagradável para a população que faz uso do Parque, constitui um forte fator de degradação dos recursos hídricos e da fauna aquática do banhado. Quanto a isso, cabe mencionar que a eutrofização do banhado existente no local ocasionou o desaparecimento da quase totalidade de espécies de peixes que ali possam ter existido, além de comprometer a sanidade dos quelônios ali observáveis.

Associado a esta possível contaminação hídrica, e considerando o quadro sintomático expresso pelos vegetais, infere-se uma significativa alteração do $\mathrm{pH}$ do solo nas áreas de influência, bem como a possível presença de elementos, traços considerados tóxicos à flora.

A acidez dos solos promove a disponibilização de elementos tóxicos para as plantas causando a diminuição da disponibilidade de nutrientes para as mesmas. Assim, por exemplo, a presença de alumínio ocasiona a redução do crescimento e o desenvolvimento das raízes e diminui a absorção de nutrientes, o que é desfavorável ao desenvolvimento de plantas sensíveis a esse elemento. Conforme Miguel et al. (2010), em solos ácidos, um fator relevante é a alta solubilidade de metais pesados, e o $\mathrm{Al}$ se avoluma por prevalecer nas soluções presentes nesse tipo de ambiente e toxidez aos vegetais. As raízes 
apresentam engrossamento e amarelamento nas pontas, degeneradas, tortuosas, com ramificações secundárias, escuras em parte pela oxidação de compostos fenólicos e sem pelos absorventes, o que parece estar em consonância com alguns espécimes que foram localizados tombados próximo às margens do arroio, com parte das raízes expostas.

\section{Conclusões}

Os resultados obtidos por este diagnóstico ambiental permitem o estabelecimento de planos estratégicos direcionados à recuperação dos arroios, ou a sua canalização pelo interior do Parque, os quais, focando características individualizadas de intervenção, permitem a seleção das melhores estratégias a serem empregadas em cada caso. A canalização, enquanto intervenção, não ocasionaria impactos sobre a fauna e flora aquáticos, visto que os registros qualitativos foram considerados para a área de banhado e não para os arroios. Além disso, as águas contaminadas dos arroios, por ocasião de enchentes, deixariam de ser intervenientes negativos sobre as espécies associadas ao banhado.

Para a recuperação da vegetação, recomenda-se a substituição gradual dos eucaliptos por espécies arbóreas nativas e o isolamento de algumas áreas com vistas a sua recuperação natural, o que igualmente favorecerá a avifauna e os Chiroptera ocorrentes na área.

Outro aspecto importante guarda relação com a presença de espécies de fauna consideradas sinantrópicas, potencialmente hospedeiras de várias zoonoses, que podem representar algum perigo para as pessoas que frequentam o Parque. Assim, novas pesquisas devem ser conduzidas com o intuito de se fazer controle e manejo dessas populações.

Finalmente, faz-se necessário também o desenvolvimento de ferramentas de sensibilização e transformação que promovam mudanças de conduta na comunidade do entorno, no sentido de possibilitar a percepção de problemas ambientais, fazendo com que cada pessoa assuma a sua responsabilidade perante o meio em que está inserida.

\section{Referências}

BACKES, Paulo; IRGANG, Bruno. Árvores do Sul: Guia de Identificação \& Interesse Ecológico. Incertae: Santa Cruz do Sul: Instituto Souza Cruz, 2002. 328p.

BACKES, Paulo; IRGANG, Bruno. Árvores Cultivadas no Sul do Brasil: Guia de Identificação e Interesse Paisagístico das Principais Espécies Exóticas. Incertae: Santa Cruz do Sul: Instituto Souza Cruz, 2004. 203p.

BAKER, P. J.; ANSELL, R. J.; DODDS, P. A. A.; WEBBER,
C. E.; HARRIS, S. Factors affecting the distribution of small mammals in an urban area. Mammal Review, Hoboken, v. 33, n. 1, p. 95-100, 2003.

BORGES-FILHO, E.M.S.; PIRES, L.P.; DEL-CLARO, K.; UIEDA, W. Comunidade de morcegos em um parque urbano de Uberlândia, Minas Gerais. In: X CONGRESSO DE ECOLOGIA DO BRASIL, 2011, São Lourenço. Anais... São Lourenço, MG: SEB. CDROM. 2011.

BORTOLETO, S. Inventário quali-quantitativo da arborização viária da Estância de Águas de São PedroSP. 2004. 85f. Dissertação (Mestrado em Agronomia) - Escola Superior de Agricultura "Luiz de Queiroz", Piracicaba, 2004.

BUJES, Clóvis S. Os Testudines continentais do Rio Grande do Sul, Brasil: taxonomia, história natural e conservação. Iheringia, Sér. Zool., Porto Alegre, v. 100, n. 4, p. 413-424, 2010.

BURBRIDGE, A. A.; MANLY, B. F. Mammals extinctions on Australian islands: causes and conservation implications. Journal of Biogeography, Malden, v. 29, n. 4, p. 465-473, 2002.

CAZETTA, E.; GALETTI, M. Ecologia das ervas-depassarinho. Ciência Hoje, Rio de Janeiro, v. 33, n. 194, p. 72-74, 2003.

EFE, Márcio A.; MOHR, Leonardo V. Projeto Ecologia e Distribuição da Avifauna dos Parques de Porto Alegre. 1999. 69 f. Relatório Técnico Final. Porto Alegre: PROAVES, CEMAVE, 1999. 69p.

EFE, Márcio A.; MOHR, Leonardo V.; BUGONI, Leandro. Guia Ilustrado das Aves dos Parques de Porto Alegre. Porto Alegre: PROAVES, SMAM, COPESUL, CEMAVE, 2001. 144p.

ENDRIGO, Edson. Aves do Pampa. São Paulo: Aves \& Fotos Editora, 2010. 224p.

FABRES, Laura; BUJES, Clóvis S. VERRASTRO, Laura. s/d. A fauna de quelônios do Parque Moinhos de Vento como ferramenta para ponderar sobre a percepção ambiental de seus usuários, Porto AlegreRS, Brasil. Disponível em: <http://www.lume. ufrgs.br/bitstream/handle/10183/49241/000835802. pdf? sequence $=1 \&$ locale $=$ pt_BR $>$. Acesso em 27 maio 2014.

FILHO, J.A. de L.; MEDEIROS, M.A.S. Impactos adversos na avifauna causados elas atividades de arborização urbana. Revista de Biologia e Ciências da Terra, Campina Grande, v. 6, n. 2, p. 375-390, 2006. 
GOMES, Cleida M. da C. F. (Coorda.). Cadastro Fotográfico da Vegetação de Porto Alegre. Porto Alegre: Secretaria Municipal do Meio Ambiente, 2011. 430p.

GUZZO P. 2006. Áreas verdes urbanas. Disponível em: $<$ http://www.educar.sc.usp.br/biologia/prociencias/ areasverdes.html>. Acesso em 22 maio 2014.

INSTITUTO BRASILEIRO DO MEIO AMBIENTE E DOS RECURSOS NATURAIS RENOVÁVEIS - IBAMA. Portaria n. 37-N, de 3 de abril de 1992. Lista Oficial de Espécies da Flora Brasileira Ameaçada de Extinção.

ISERNHAGEN, I.; BOULERGAT, J.M.G.; CARBONI, $\mathrm{M}$. Trazendo a riqueza arbórea regional para dentro das cidades: possibilidades, limitações e benefícios. Revista da Sociedade de Arborização Urbana, Piracicaba, v. 4, n. 2, p.117-138, 2009.

JAPYASSÚ, H. F.; BRESCOVIT, A. Biodiversidade araneológica na cidade de São Paulo: a urbanização afeta a riqueza de espécies? 3p. Disponível em: <www. ambientebrasil.com.br>. Acesso em 23 maio 2014.

KENT, M.; COKER, P. Vegetation description and analysis: a practical approach. New York: John Wiley \& Sons, 1992. 363p.

LEAL, L.; BUJOKAS, W. M.; BIONDI, D. Analise da infestação de erva-de-passarinho na arborização de ruas de Curitiba, PR. Revista Floresta, Curitiba, v. 36, n. 3, p. 323-330, 2006.

LEPCZYK, C. A.; MERTIG, A. G.; LIU, J. Landowners and cat predation across rural-to-urban landscapes. Biological Conservation, London, v. 115, n. 2, p. 191201, 2003.

LOMBARDO, M. A. Vegetação e clima. In: III ENCONTRO NACIONAL SOBRE ARBORIZAÇÃO URBANA. Anais... Curitiba, PR. p. 01 -13. 1990.

LONGHI, Rubens A. Livro das árvores: árvores e arvoretas do Sul. Porto Alegre: L\&PM, 1995. 176p.

LORENZI, Harri. Árvores Brasileiras: Manual de Identificação e Cultivos de Plantas Arbóreas Nativas do Brasil. Nova Odessa: Instituto Plantarum, 2002. 384p.

LORENZI, Harri; SOUZA, Hermes M. de. Plantas Ornamentais no Brasil: arbustivas, herbáceas e trepadeiras. Nova Odessa: Instituto Plantarum, 1995. 736 .

LORENZI, Harri; SOUZA, Hermes M. de; TORRES, Mario A. V.; BACHER, Luis B.. Árvores Exóticas no Brasil: madeireiras, ornamentais e aromáticas. Nova
Odessa: Instituto Plantarum, 2003. 384p.

MACEDO S.S., SAKATA F.G. Parques Urbanos no Brasil. 2a ed. EDUSP/ Imprensa Oficial do Estado de São Paulo: São Paulo, 2003. 208p.

MACIEL, Renata P. Revisão do status taxonômico de Amphisbaena prunicolor (Cope, 1885) e Amphisbaena albocingulata Boettger, 1885 (Amphisbaenia: Amphisbanidae). 2011. 80f. Dissertação (Mestrado em Biologia Animal) - Programa de Pós Graduação em Biologia Animal, Instituto de Biociências, UFRGS, Porto Alegre, 2011.

MARCHIORI, José N. C. Dendrologia das Gimnospermas. Santa Maria: Ed. da UFSM, 1996. 158p.

MENEGAT, R.; PORTO, M. L.; CARRARO, E. C.; BERNARDES, L. Atlas Ambiental de Porto Alegre. Porto Alegre: Ed. da Universidade/ UFRGS, 1998. 228p.

MIGUEL, Paulo S. B.; GOMES, Fernando T.; ROCHA, Wadson S. D. da; MARTINS, Carlos E.; CARVALHO, Caio A. de; OIIVEIRA, André V. de. Efeitos tóxicos do alumínio no crescimento das plantas: mecanismos de tolerância, sintomas, efeitos fisiológicos, bioquímicos e controles genéticos. Centro de Ensino Superior Revista, Juiz de Fora, v. 24, p. 13-29, 2010.

OLIVEIRA, F. B.; KAPPEL, R. B.; Incidência de ervas de passarinho na arborização de ruas de Porto Alegre. In: Congresso Brasileiro de Arborização Urbana, 2, 1994, São Luís, Anais... São Luís, 1994. p. 335- 346.

PACHECO, S. M.; MARQUES, R. V. Conservação de morcegos no Rio Grande do Sul. In: FREITAS, T; VIEIRA, E.; PACHECO, S; CHRISTOFF, A. (Org.). Mamíferos do Brasil: Genética, Sistemática, Ecologia e Conservação. São Carlos: Ed. dos organizadores, 2006. p. 91-106.

PARDINI, R.; DITT, E. H.; CULLERJR, L.; BASSI, C.; RUDRAN, R. Levantamento rápido de mamíferos terrestres de médio e grande porte. In: CULLENJR, L.; RUDRAN, R.; VALLADARES-PADUA, C. (Orgs). Métodos de estudo em biologia da conservação e manejo da vida silvestre. Curitiba: Editora da Universidade Federal do Paraná, 2003. 665p.

PENTER, Camila; PEDÓ, Ezequiel; FABIÁN, Marta E.; HARTZ, Sandra M. Inventário Rápido da Fauna de Mamíferos do Morro Santana, Porto Alegre, RS. Revista Brasileira de Biociências, Porto Alegre, v. 6, n. 1, p. 117-125, 2008.

PUENTE, André D.; TORRES, Vladimir S.; BLEICKER, Paola P. Floresta urbana e biodiversidade. In: IX 


\section{CONGRESSO BRASILEIRO DE ARBORIZAÇÃO} URBANA, Anais do... Belo Horizonte, 2005. Disponível em: <http://lproweb.procempa.com.br/pmpa/prefpoa/ smam/usu_doc/floresta_urbana_e_biodiversidade. pdf $>$. Acesso em:16 maio 2014.

RIO GRANDE DO SUL. Lei № 9.519, de 21 de janeiro de 1992. Institui o Código Florestal do Estado do Rio Grande do Sul e dá outras providências.

SCHNEIDER, P. R. Manejo Florestal: Planejamento da Produção Florestal. Santa Maria: CEPEF/FATEC/ UFSM. 2002. 492p.

SENNA, R.M.; KAZMIRCZAK, C. Pteridófitas de um remanescente florestal no Morro da Extrema, Porto Alegre, RS. Revista Faculdade Zootecnia, Veterinária e Agronomia, Uruguaiana, v. 4, n. 1, p. 47-57, 1997.

SILVA, Flávio. Mamíferos Silvestres do Rio Grande do Sul. Porto Alegre: Fundação Zoobotânica do Rio Grande do Sul, 1994. 246p.

SILVA, Rafael I. da; SILVA, Hellen R. A. da; ARAÚJO, Bruno de S.; CARREGARO, Juliano B. Composição e comportamento da avifauna de um parque urbano em Brasília, Distrito Federal. In: X CONGRESSO DE ECOLOGIA DO BRASIL, 2011, São Lourenço. Anais... São Lourenço, MG: SEB. CD-ROM. 2011.

SOBRAL, Marcos; JARENKOW, João A.; BRACK, Paulo; IRGANG, Bruno; LAROCCA, João; RODRIGUES, Rodrigo S. Flora Arbórea e Arborescente do Rio Grande do Sul, Brasil. São Carlos: RiMa: Novo Ambiente, 2006. 350p.

TORRES, Vladimir S. Contribuição à biologia de amphisbaenidae (Reptilia: Amphisbaenia) encontradas em Porto Alegre, RS, Brasil. Notes fauniques de Gembloux, Gembloux v. 53, p. 63-69, 2003.

WITT, Patrícia B. R. (Coorda.). Fauna e Flora da Reserva Biológica do Lami José Lutzenberger. Porto Alegre: Secretaria Municipal do Meio Ambiente, 2013. 308p.

WOODS, M.; MCDONALD, R.; HARRIS, S. 2003. Predation of widlife by domestic cat Felis catus in Great Britain. Mammal Review, v. 33, n. 1, p. 174-188, 2003.

WRIGHT, S. J.; ZEBALLOS, H.; DOMINGUEZ, I.; GALLARDO, M.; MORENO, M. C.; IBÁÑEZ, R. Poachers alter mammal abundance, seed dispersion and seed predation in a neotropical forest. Conservation Biology, Germantown, v. 14, n. 1, p. 227-239, 2000.

YOUNG, B.; SEDAGHATKISH, G.; ROCA, R. Levantamentos de fauna. In: SAYRE, R.; ROCA, E.;
SEDAGHATKISH, G.; YOUNG, B.; KEEL, S.; ROCA, R.; SHEPPARD, S. (Org.). Natureza em Foco: Avaliação Ecológica Rápida. Arlington: The Nature Conservancy, 2003. 194p. 